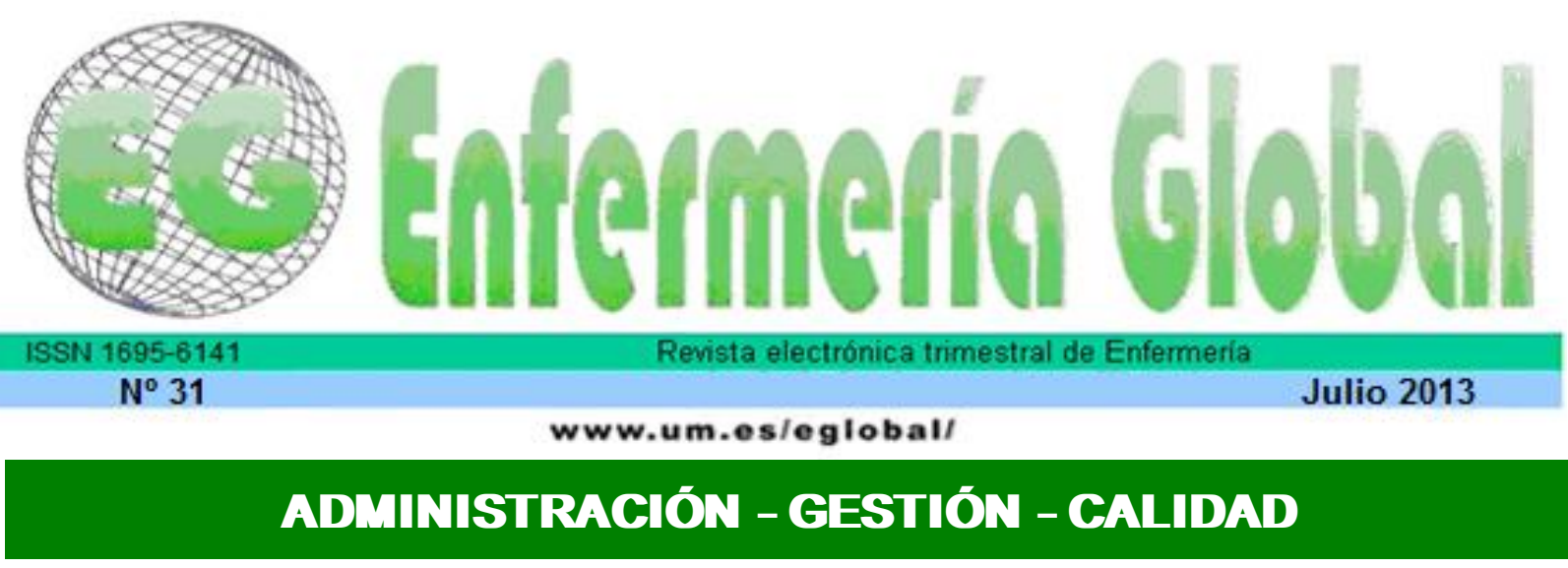

\title{
Percepción del estrés en los estudiantes de Enfermería ante sus prácticas clínicas
}

Stress perception in Nursing students facing their clinical practices

\section{*Moya Nicolás, María *Larrosa Sánchez, Sandra *López Marín, Cristina *López Rodríguez, Irene *Morales Ruiz, Lidia *Simón Gómez, Ángela}

\author{
*Enfermera. Universidad de Murcia. España. E-mail: maria.moya3@um.es \\ Palabras clave: estrés; estudiantes de Enfermería; prácticas clínicas \\ Keywords: Stress; Nursing students; clinical practices
}

\section{RESUMEN}

Objetivo. Esta investigación pretende conocer cuáles son las principales fuentes de estrés durante el periodo de prácticas de los estudiantes de Enfermería de la Universidad de Murcia en función de su sexo, curso y edad.

Metodología. Se trata de un estudio transversal de corte descriptiva. La muestra estuvo compuesta por un total de 45 alumnos, 30 mujeres y 15 hombres, de Enfermería de la Universidad de Murcia del curso académico 2010/2011. El instrumento de recogida de la información fue cuestionario KEKZAK.

Resultados. El desconocimiento ante una determinada situación clínica y el riesgo de dañar al paciente son las dos principales fuentes estresoras para los estudiantes de enfermería. Los hombres suelen sufrir un mayor estrés que sus compañeras; no obstante, a medida que aumenta la edad y se avanza de curso el grado de estrés es cada vez menor.

Conclusiones. El estrés de los estudiantes de enfermería marca su formación durante el período de prácticas clínicas. Por tanto, se hace necesario que el aprendizaje teórico que sustenta a las prácticas atienda a estas necesidades que los propios estudiantes de enfermería demandan.

\section{ABSTRACT}

Objective: This research aims to establish what the Nursing students' main stress sources are during their practice period, according to their gender, grade and age.

Methodology. It is a descriptive transversal study. The sample was composed by 45 Nursing students, 30 women and 15 men, at the University of Murcia in 2010/2011. The information gathering tool was KEZKAK questionnaire.

Results. The ignorance facing a determined clinical situation and the risk of damaging the patient are the Nursing students' two main stress sources. Men often suffer from a bigger stress than women; nevertheless, as well as the age increases and the students pass grades, the stress level is smaller. 
Conclusions. Nursing students' stress determines their formation within their clinical practices period. Thus, it is necessary that the theoretical learning that supports these practices considers theses needs that own Nursing students require.

\section{INTRODUCCIÓN}

Durante el proceso de vida, el ser humano busca adaptarse continuamente a los cambios que le ocurren, tratando de lograr el equilibrio entre su organismo y el medio ambiente, lo que origina una respuesta al estrés necesaria para afrontar nuevas situaciones ${ }^{(1,2,3)}$.

Actualmente el estrés y su estudio han alcanzado gran popularidad tanto en el lenguaje cotidiano como en la literatura científica, siendo considerado como un factor predisponente, desencadenante o coadyuvante de múltiples enfermedades, las cuales pueden de gravedad ${ }^{(4)}$.

El vocablo estrés fue acuñado por el fisiólogo Hans Selye, quien en 1950 lo definió como "una reacción del organismo frente a las demandas del entorno" (5).

Desde 1950 se ha conceptualizado el estrés de distintas formas, bien como respuesta, como estímulo o como proceso ${ }^{(5,6)}$. Así Holmes y Rahe entienden el estrés como: "un estímulo variable independiente o carga que se produce sobre el organismo, generando malestar, de tal forma que si se sobrepasan los límites de tolerancia, el estrés comienza a ser insoportable, apareciendo entonces los problemas psicológicos y físicos" ${ }^{(7)}$.

Por otro lado, Lazarus y Folkman, desde el enfoque del estrés como proceso, lo definen como: "una relación transicional entre la persona y el entorno que es evaluado por ésta como amenazante o desbordante de sus recursos y que pone en peligro su homeostasis y bienestar"; dándose así una interacción dinámica de dos procesos básicos: la valoración cognoscitiva que cada persona hace sobre las demandas ambientales y las estrategias de afrontamiento con que cuenta para hacer frente a esas demandas ${ }^{(8)}$.

Actualmente, el estrés está considerado como el resultado de la incapacidad del individuo de hacer frente a las demandas del ambiente, a diferencia de la ansiedad que sería la reacción emocional ante una amenaza manifestada a nivel cognitivo, fisiológico, motor y emocional ${ }^{(9)}$. De modo que la ansiedad constituye la principal respuesta emocional asociada al estrés; considerándolo una condición transitoria, caracterizada por sentimientos subjetivos de tensión, aprensión, inquietud y preocupación, así como una hiperactividad del sistema nervioso autónomo, la cual puede variar en intensidad y fluctuar en el tiempo ${ }^{(10)}$.

A lo largo de nuestra vida todos hemos experimentado alguna vez las consecuencias negativas que produce el estrés. Del mismo modo, durante la vida académica, los estudiantes y en concreto aquellos que llevan a cabo prácticas en los centros hospitalarios como los alumnos de enfermería, están expuestos a los efectos propios de una sintomatología del denominado estrés ${ }^{(11)}$. Asimismo, debemos partir de la premisa de que "lo que es considerado estresante para unos no lo es para otros", por tanto el estrés tendrá un alcance distinto según el estudiante. En consecuencia, este hecho origina que los estudiantes en un intento de adaptarse a estas situaciones pongan en marcha una serie de estrategias de afrontamiento para superar con éxito 
las exigencias que se les demanda, buscando el alivio de su estado tensional. Sin embargo, una gran parte de estudiantes universitarios carecen de estrategias 0 adoptan estrategias inadecuadas ${ }^{(12)}$.

Las prácticas clínicas son un elemento fundamental en la formación de los estudiantes de enfermería, ya que les permiten aplicar los conocimientos teóricos y desarrollar habilidades para la prestación de cuidados a los pacientes (13) Las prácticas hospitalarias tienen un gran impacto educativo ya que los conocimientos que se adquieren en ellas permiten el desarrollo de las habilidades y actitudes propias de cada estudiante que les son necesarias para la adquisición de competencias y les permiten incorporarse como profesionales en el ámbito sanitario ${ }^{(14)}$.

En las prácticas clínicas, los alumnos toman contacto con su futura profesión y se entrenan en la misma, enfrentándose a situaciones difíciles, que en muchas ocasiones son generadoras de estrés y no siempre se realizan en un entorno favorecedor que les permite entender la practica enfermera en su totalidad ${ }^{(10)}$.

El entorno sanitario suele proporcionar muchos estímulos generadores de estrés debido al contacto con las enfermedades, el dolor, el sufrimiento, la invalidez, la muerte de los pacientes, la necesidad de establecer relaciones con diversos profesionales sanitarios y pacientes, así como el hecho de tener que desempeñar un nuevo rol para el que aún no están completamente preparados ${ }^{(15)}$.

En diversos estudios se han descrito las principales fuentes de estrés en los estudiantes de enfermería, asociándolos con el área clínica y académica, sin olvidar otras áreas presentes como son lo social e interpersonal (16). Zryewsky y Davis comprobaron que las áreas, académica y clínica generaban el $78,4 \%$ de los acontecimientos estresantes, siendo el área social el $8 \%$ y el área personal el 13,6\% (17)

Entre las situaciones vividas con más intensidad por los estudiantes de enfermería durante las prácticas asistenciales se encuentran: la muerte del paciente, el sufrimiento, el contacto con enfermos terminales y el afrontamiento de la muerte, las situaciones de patología crítica, el paro cardiorrespiratorio, la relación con el enfermo y su familia y la diferencia entre la teoría y la práctica. No obstante, las relaciones con los profesionales, los sentimientos de impotencia y la falta de conocimientos y destreza en las técnicas son los aspectos que más preocupan a los alumnos de enfermería ${ }^{(13)}$. Timmins y Kaliszer han observado que las principales fuentes de estrés en el último curso de enfermería son: el estrés académico, la relación con los profesores, las relaciones en las prácticas clínicas, las finanzas y la muerte de los pacientes ${ }^{(18,19)}$.

Por ello, en la formación de los estudiantes de enfermería es fundamental conocer las fuentes de estrés relacionadas con el rol que desempeñan durante las prácticas para determinar qué habilidades potenciar y así contribuir a la salud laboral de los futuros profesionales e, indirectamente, a un mejor cuidado de las personas atendidas (9). Según la bibliografía recogida, hasta el año 2003 no existía ningún cuestionario, ni en castellano ni en inglés, referenciado que nos sirviera para medir las fuentes de estrés en la práctica clínica de los estudiantes ${ }^{(18)}$.

En consecuencia, nuestra investigación recoge como objetivo principal conocer cuáles son las principales fuentes de estrés en la práctica clínica desde el punto de vista de 
los estudiantes de enfermería, así como su evolución a lo largo de la diplomatura y, más concretamente, medir los estresores correspondientes a cada curso académico y determinar con ello los más predominantes, determinar el nivel de estrés por: edad, sexo y curso y su relación con el nivel de estrés, conocer las situaciones que resultan más estresantes para los estudiantes y comparar las posibles diferencias entre los cursos.

Además, interesa conocer la evolución de esas fuentes de estrés a medida que el alumno se forma y va adquiriendo experiencia con el fin de prevenir situaciones estresantes. El trabajo de enfermería genera estrés por aspectos relacionados con el trabajo en sí (rol) y por aspectos derivados de la organización del trabajo ${ }^{(20)}$.

\section{METODOLOGÍA}

Los participantes fueron 45 alumnos (15 hombres y 30 mujeres) con edades comprendidas entre los 17 y los 24 años de edad $(M=19,67 ; S D=1,85)$ matriculados en la Diplomatura en Enfermería de la Universidad de Murcia en el año 2011. Se incluyó a 16 alumnos de primer curso, a 15 alumnos de segundo curso y a 14 alumnos de tercer curso. Las variables sociodemográficas consideradas han sido el sexo (hombre y mujer), el curso académico en el que se encuentran matriculados (primero, segundo o tercero) y la edad en años. El método de muestreo utilizado fue no probabilístico por conveniencia participando aquellos alumnos que voluntariamente quisieron hacerlo.

Se trata de un estudio de tipo transversal y de corte descriptiva ${ }^{(21)}$ que utiliza como herramienta el cuestionario KEZKAK ${ }^{(18)}$, que mide factores causantes de estrés en las prácticas clínicas de alumnos de enfermería, presentando un alto nivel de validez y fiabilidad ${ }^{(22,23)}$. Consta de 12 ítems a contestar mediante una escala tipo Likert de 4 valores que comprende desde (0) "nada estresante" hasta (3) "muy estresante".

El análisis de los datos se realizó con el paquete estadístico SPSS 20. La significación estadística se consideró con aquellos valores cuya probabilidad fue inferior a p =.050.

\section{RESULTADOS}

Los datos descriptivos del total de la muestra para cada una de las variables estresoras tratadas se presentan en la tabla 1. 
Tabla 1. Estadísticos descriptivos de las fuentes estresoras

\begin{tabular}{|c|c|c|}
\hline & Media & $\begin{array}{c}\text { Desv. } \\
\text { típ. }\end{array}$ \\
\hline Manejo de las Nuevas Tecnologías & 3,49 & 1,24 \\
Desconocimiento ante Situación Clínica & 4,51 &, 92 \\
Contacto con el Sufrimiento Ajeno & 3,62 & 1,21 \\
Relaciones con los profesionales en las \\
Prácticas & 3,73 & 1,36 \\
Impotencia e Incertidumbre ante Situaciones & 4,40 &, 84 \\
No Saber Controlar las Relaciones con el & 2,67 & 1,35 \\
Paciente & 4,02 & 1,32 \\
Implicaciones Emocionales con el Enfermo & 1,12 \\
Riesgo a Sufrir Contagio, Daños o Lesiones & 4,27 &, 82 \\
Riesgo a Dañar al Paciente & 4,51 & 1,42 \\
Relaciones con Familiares del Paciente & 2,29 &, 98 \\
Exceso - Sobrecarga de Trabajo & 4,16 & 1,08 \\
Resultado de la Evaluación de las Prácticas & 3,58 &
\end{tabular}

Se pone de manifiesto que las principales situaciones causantes de estrés son el desconocimiento ante una determinada situación clínica, la impotencia e incertidumbre ante éstas y el riesgo a causar un daño sobre el paciente. Se trata de tres variables relacionadas con la calidad de la formación, especialmente aquella de carácter práctico que parece ser inadecuada o, como mínimo, insuficiente, de acuerdo con la opinión mostrada por los propios estudiantes de enfermería.

En el extremo opuesto, las relaciones sociales, tanto con el paciente como con sus familiares se han mostrado como las fuentes de estrés menos potentes de entre las estudiadas.

A continuación se analiza la relación que puede existir entre la variable sexo y la variable curso con cada una de las fuentes estresoras. Para ello, el análisis de la normalidad de ambas variables mediante la prueba de $\chi^{2}$ obteniendo una distribución no paramétrica para el sexo y paramétrica para el curso. Por tanto, para la correlación entre el sexo y las fuentes de estrés se ha utilizado la prueba estadística $U$ de Mann Whitney alcanzando la significación estadística en 4 de las 12 fuentes estresoras. En la tabla 2 se muestran la significatividad de estas relaciones y los valores para cada sexo.

Tabla 2. Correlación entre el sexo y las fuentes estresoras

\begin{tabular}{|c|c|c|c|}
\hline Fuente de estrés & \multicolumn{2}{|c|}{ Sexo } & \multirow{2}{*}{ Sig. } \\
\cline { 2 - 4 } & Hombre & Mujer &, 001 \\
\hline $\begin{array}{c}\text { Manejo de las } \\
\text { Nuevas Tecnologías }\end{array}$ & $4,33 \pm 0,82$ & $3,07 \pm 1,22$ &, 245 \\
\hline $\begin{array}{c}\text { Desconocimiento } \\
\text { ante Situación Clínica }\end{array}$ & $4,33 \pm 0,98$ & $4,60 \pm 0,89$ &, 000 \\
\hline
\end{tabular}




\begin{tabular}{|c|c|c|c|}
\hline $\begin{array}{l}\text { Contacto con el } \\
\text { Sufrimiento Ajeno }\end{array}$ & $4,53 \pm 0,64$ & $3,17 \pm 1,18$ & ,000 \\
\hline $\begin{array}{l}\text { Relaciones con los } \\
\text { profesionales en las } \\
\text { Prácticas }\end{array}$ & $3,80 \pm 1,42$ & $3,70 \pm 1,34$ & ,753 \\
\hline $\begin{array}{c}\text { Impotencia e } \\
\text { Incertidumbre ante } \\
\text { Situaciones }\end{array}$ & $4,53 \pm 0,52$ & $4,33 \pm 0,96$ & ,860 \\
\hline $\begin{array}{l}\text { No Saber Controlar } \\
\text { las Relaciones con el } \\
\text { Paciente }\end{array}$ & $3,80 \pm 1,01$ & $2,10 \pm 1,13$ & ,000 \\
\hline $\begin{array}{c}\text { Implicaciones } \\
\text { Emocionales con el } \\
\text { Enfermo }\end{array}$ & $4,27 \pm 0,96$ & $3,90 \pm 1,47$ &, 559 \\
\hline $\begin{array}{c}\text { Riesgo a Sufrir } \\
\text { Contagio, Daños o } \\
\text { Lesiones }\end{array}$ & $4,40 \pm 0,83$ & $4,20 \pm 1,24$ & 901 \\
\hline $\begin{array}{l}\text { Riesgo a Dañar al } \\
\text { Paciente }\end{array}$ & $4,60 \pm 0,63$ & $4,47 \pm 0,90$ & ,776 \\
\hline $\begin{array}{c}\text { Relaciones con } \\
\text { Familiares del Paciente }\end{array}$ & $2,40 \pm 1,50$ & $2,23 \pm 1,41$ & ,749 \\
\hline $\begin{array}{c}\text { Exceso - } \\
\text { Sobrecarga de Trabajo }\end{array}$ & $4,33 \pm 0,90$ & $4,07 \pm 1,02$ & ,379 \\
\hline $\begin{array}{c}\text { Resultado de la } \\
\text { Evaluación de las } \\
\text { Prácticas }\end{array}$ & $4,20 \pm 1,01$ & $3,27 \pm 0,98$ & ,007 \\
\hline
\end{tabular}

De este modo queda latente el mayor estrés experimentado por los hombres, frente a las mujeres, en las categorías de manejo de las nuevas tecnologías, contacto con el sufrimiento ajeno, no saber controlar las relaciones con el paciente y la preocupación por el resultado de la evaluación de las prácticas.

Para el estudio del vínculo entre las situaciones estresantes y el curso se ha utilizado la prueba estadística ANOVA, obteniendo como resultado la tabla 3.

Tabla 3. Correlación entre el curso y las fuentes estresoras

\begin{tabular}{|c|c|c|c|c|c|c|}
\hline & & $\begin{array}{l}\text { Suma de } \\
\text { cuadrados }\end{array}$ & $1^{g}$ & $\begin{array}{r}\text { Media } \\
\text { cuadrática }\end{array}$ & $\mathrm{F}$ & $\begin{array}{l}\text { S } \\
\text { ig. }\end{array}$ \\
\hline \multirow[t]{3}{*}{$\begin{array}{l}\text { Manejo de las Nuevas } \\
\text { Tecnologías }\end{array}$} & $\begin{array}{l}\text { Inter- } \\
\text { grupos }\end{array}$ & 22,378 & 2 & 11,189 & $\begin{array}{r}10,4 \\
74\end{array}$ & 000 \\
\hline & $\begin{array}{l}\text { Intra- } \\
\text { grupos }\end{array}$ & 44,866 & $2^{4}$ & 1,068 & & \\
\hline & Total & 67,244 & $\begin{array}{r}4 \\
4\end{array}$ & & & \\
\hline \multirow[t]{3}{*}{$\begin{array}{l}\text { Desconocimiento ante } \\
\text { Situación Clínica }\end{array}$} & $\begin{array}{l}\text { Inter- } \\
\text { grupos }\end{array}$ & 1,347 & 2 & ,673 & ,788 & 461 \\
\hline & & 35,898 & $2^{4}$ & ,855 & & \\
\hline & Total & 37,244 & $4^{4}$ & & & \\
\hline \multirow[t]{3}{*}{$\begin{array}{l}\text { Contacto con el Sufrimiento } \\
\text { Ajeno }\end{array}$} & $\begin{array}{l}\text { Inter- } \\
\text { grupos }\end{array}$ & 19,644 & 2 & 9,822 & $\begin{array}{r}9,18 \\
1\end{array}$ & 000 \\
\hline & $\begin{array}{l}\text { Intra- } \\
\text { grupos }\end{array}$ & 44,933 & $2^{4}$ & 1,070 & & \\
\hline & Total & & $4^{4}$ & & & \\
\hline \multirow[t]{3}{*}{$\begin{array}{l}\text { Relaciones con los } \\
\text { Profesionales en las Prácticas }\end{array}$} & $\begin{array}{l}\text { Inter- } \\
\text { grupos }\end{array}$ & 1,272 & 2 & ,636 & ,336 & 717 \\
\hline & $\begin{array}{l}\text { Intra- } \\
\text { grupos }\end{array}$ & 79,528 & $2^{4}$ & & & \\
\hline & Total & & $4^{4}$ & & & \\
\hline
\end{tabular}




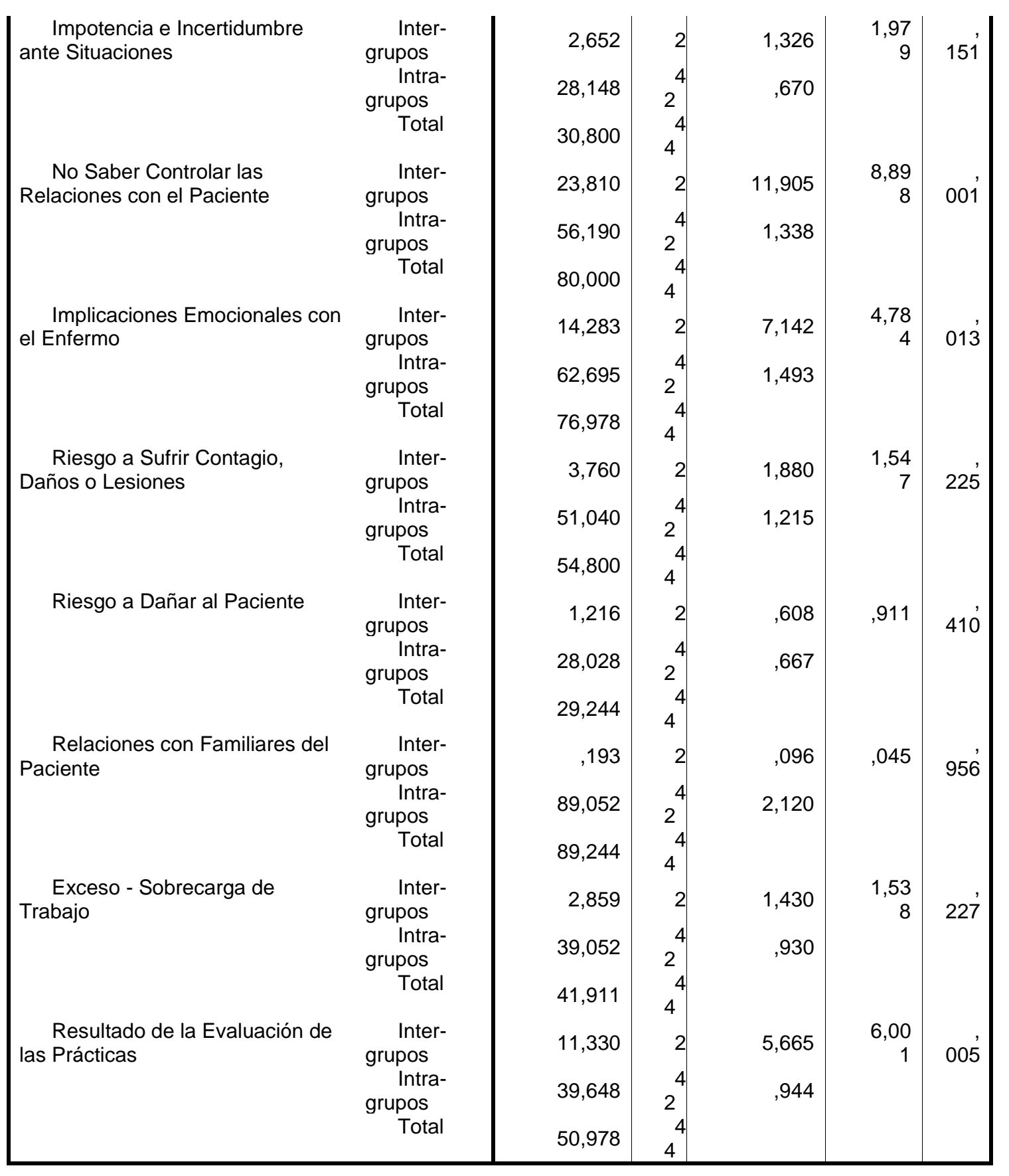

De acuerdo con los datos expuestos, se aprecian diferencias significativas para 5 fuentes de estrés, a saber: el manejo de las nuevas tecnologías, el contacto con el sufrimiento ajeno, el no saber controlar las relaciones con el paciente, las implicaciones emocionales que se puedan contraer con éste y la preocupación por la calificación de sus prácticas. La evolución del grado de desarrollo de estas variables para cada uno de los cursos se muestra en la figura 1. 
Figura 1. Evolución de las fuentes estresoras con cambios significativos

$\mathrm{Se}$

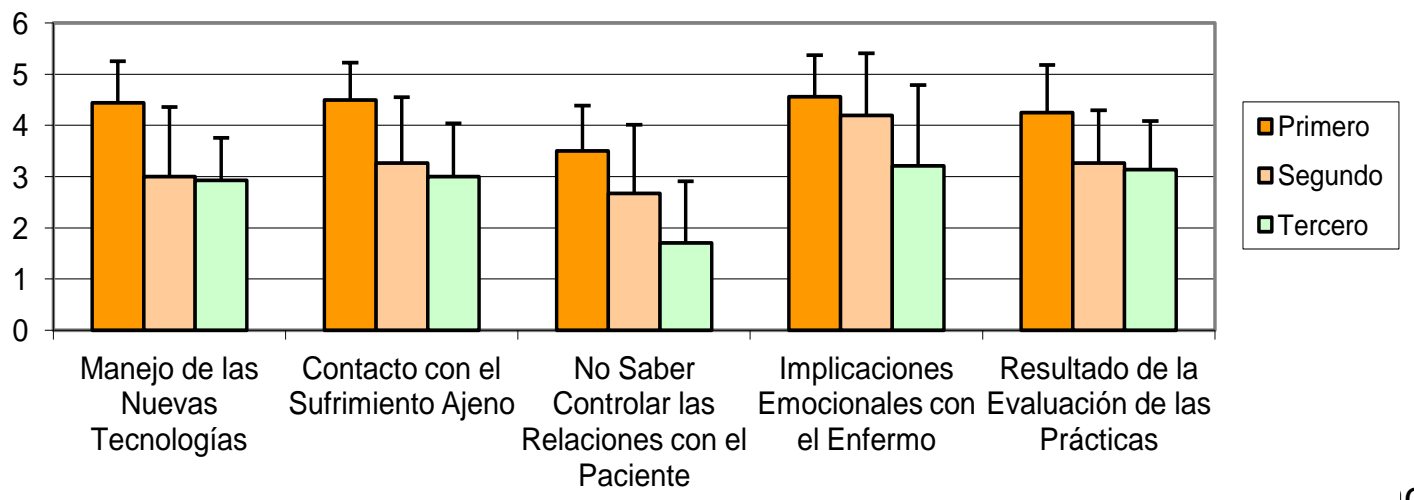

estresantes en la medida en la que su grado de desarrollo es menor conforme los alumnos avanzan en la Diplomatura en Enfermería.

Finalmente, con respecto a la edad de los participantes, se ha realizado la prueba Kolmogorov-Smirnov, resultando una distribución de los datos no paramétrica; por tanto, para el estudio de su relación bivariada con cada una de las fuentes estresoras se ha recurrido al análisis de la Correlación de Pearson.

Dado que los alumnos de cursos más avanzados suelen tener mayor edad que los de los primeros cursos, la significación estadística y con el mismo signo para su evolución (reducción conforme aumenta la edad) se ha alcanzado para las mismas fuentes estresoras que se presentaban para el análisis con el curso, esto es: manejo de las nuevas tecnologías $(r=-, 365 p=, 014)$, contacto con el sufrimiento ajeno $(r=-$ ,352 $p=, 018)$, no saber controlar las relaciones con el paciente $(r=-, 511 p=, 000)$, las implicaciones emocionales con el enfermo $(r=-, 313 p=, 036)$ y la preocupación por la calificación $(r=-, 313 p=, 037)$.

\section{DISCUSIÓN}

La puntuación media obtenida en la totalidad de la muestra refleja una considerable percepción del estrés en los alumnos durante la realización de las prácticas clínicas. Ello coincide con otros estudios en los que también se encontró una alta percepción del estrés en los estudiantes de enfermería en su período práctico ${ }^{(13)}$. Debemos tener en cuenta que estas puntuaciones nos informan de qué es lo que les parece más estresante a los alumnos en las prácticas clínicas, no el nivel de estrés.

Hay que destacar que un número importante de las situaciones reflejadas en el cuestionario son percibidas por los estudiantes como bastante o muy estresantes. Los aspectos hallados más estresantes para los estudiantes de enfermería por otros autores, tales como el "desconocimiento ante la situación clínica" (11) y la "impotencia e incertidumbre ante situaciones desconocidas" ${ }^{(8)}$ se corresponden con los factores más estresantes de este estudio. Sin embargo, discrepamos con respecto al factor "riesgo a dañar al paciente" ${ }^{(8)}$, el cual no aparece como elemento estresante en los alumnos de enfermería. En otros estudios se indicaron como más estresantes las situaciones referidas a las relaciones con los profesionales, compañeros, pacientes y profesores de prácticas ${ }^{(4)}$, mientras que nuestros resultados sitúan a los factores relativos a esas situaciones (factores 1,4 y 10) como los menos causantes de estrés.

En cuanto a los factores de estrés, podemos considerar que las situaciones de estrés que viven con mayor intensidad se deben a la inseguridad que representa el 
enfrentarse a situaciones nuevas y al miedo a no saber responder, más que a un rasgo de su personalidad. El hecho de tratarse de una población en formación y no tener aún la habilidad y seguridad suficientes para la prestación de cuidados puede explicar que el miedo a realizar un trabajo incorrecto y perjudicar al paciente, pincharse con una aguja infectada y contagiarse a través del paciente sean los aspectos que les produzcan más estrés, asunción que se descrita en otros estudios $^{(24)}$.

La relación entre el género y las correspondientes medias las hemos considerado por estar compuesta nuestra muestra mayoritariamente por mujeres aunque, es importante subrayar que los resultados no son representativos en cuanto al género tanto masculino como femenino, a causa del escaso número de la muestra. Pese a ello, en relación a las variables sexo y curso interconectado con los factores que producen estrés podemos apreciar que los hombres sufren en mayor medida los agentes estresantes que las mujeres, afirmación que se encuentra en contraposición de lo afirmado por los estudios de Amat, et al. ${ }^{25)}$, los cuales manifiestan que los hombres pueden desarrollar en un primer momento mayor reactividad cognitiva aunque este sentimiento desaparece a lo largo de las prácticas clínicas.

Destacamos haber encontrado una mayor percepción del estrés entre los estudiantes de primer curso que entre los de segundo y tercero, si bien la diferencia no es muy acusada en algunos factores estresantes, en otros la diferencia es enorme. Es posible que el hecho de que los alumnos de primer curso percibieran las situaciones como más estresantes se deba a un menor contacto con los pacientes y a no haber vivido de cerca esas situaciones que sus compañeros de segundo y tercero, ya que los alumnos de primero no realizan prácticas clínicas en este curso académico ${ }^{(20,22)}$. No obstante, los alumnos de tercero presentan una percepción del estrés disminuida en comparación con los otros dos grupos. En el tercer curso, estos resultados pueden estar relacionados con la mayor seguridad que les proporciona la realización de sus prácticas en un ambiente ya conocido. Tener más conocimientos por otra parte disminuye su preocupación en ítems como "Encontrarme en una situación sin saber qué hacer", "Encontrarme ante una situación de urgencia" o "Recibir órdenes contradictorias" que como sabemos, son situaciones en las que todavía no se han enfrentado los alumnos de tercer curso solos y tienen miedo a no ser capaces de resolver correctamente se encuentra en consonancia con otros estudios semejantes desarrollados por López-Medina \& Sánchez-Collado ${ }^{(13)}$

Por otro lado, al igual que Ferrer Pascual et al. ${ }^{(15)}$, se ha encontrado una ligera disminución de la percepción del estrés a mayor edad de los alumnos, aunque a diferencia de la investigación mencionada, en algunos de los factores estresantes como son: "contacto con el sufrimiento ajeno" o "resultado de la evaluación de las prácticas" existe una igualdad de los resultados.

\section{CONCLUSIONES}

Las características personales de la población en estudio fueron: sexo, predominantemente el femenino, el grupo de edad más frecuente fue de 19 años, curso académico, primero, segundo y tercero, y los factores de estrés.

Del total de la muestra estudiada, la mayoría presenta niveles de estrés severo; la fuente de estrés según el tema traumático fue el estrés producido por las prácticas 
clínicas, es así que el evento más frecuentemente reportado como estresante es el "desconocimiento ante la situación clínica".

Entre sus primeros contactos con la profesión de enfermería temen que tanto el paciente como ellos mismos resulten dañados. Por el contrario, la relación con los distintos estudiantes, profesionales, tutores y pacientes es lo que menos estrés les produce, quizá porque esto no pone en peligro la seguridad del paciente ni la suya propia y porque están más formados y capacitados para establecer relaciones sociales adecuadas que para hacer frente a las situaciones anteriores. El nivel de estrés se relaciona de manera significativa con el uso de estrategias enfocadas al problema; sin embargo, el uso de las emociones también se relaciona de manera significativa con elevados niveles de estrés. Con lo cual, llevar a cabo técnicas junto con terapias dirigidas a disminuir y afrontar el estrés producen un cambio significativo en el alumno.

Por todo ello consideramos que sería importante realizar un seguimiento longitudinal a aquellos alumnos que todavía permanecerán en la escuela durante el curso 2011/12, con la finalidad de comprobar si los niveles de percepción de las situaciones estresantes se mantienen o disminuyen a lo largo del tiempo.

Dada la trascendencia del estrés detectado en los estudiantes de enfermería durante las prácticas clínicas, consideramos que sería necesario aplicar programas de formación en estrategias para la reducción del estrés. Éstos pueden ser muy útiles para ayudar a los futuros profesionales a identificar los agentes estresores, disminuir su percepción del estrés y realizar su trabajo cotidiano disfrutándolo y sin riesgos para su salud. Las situaciones ordenadas de mayor a menor percepción de estrés en los alumnos de este estudio pueden ayudarnos a priorizar y planificar las intervenciones educativas al permitirnos conocer con mayor exactitud qué aspectos vividos en las prácticas clínicas les afectan más y cuáles son percibidos con menor intensidad. De ese modo, será más fácil que los futuros profesionales de enfermería reaccionen de una forma adaptativa a los estímulos estresantes a los que van a enfrentarse diariamente en su ámbito laboral.

Por parte de los docentes, sería importante que a la hora de la enseñanza a los alumnos, ésta fuese realizada dentro de modelos reales, ya que el estar desviado en exceso de la realidad no favorece el aprendizaje.

Recordar también a nuestros compañeros profesionales que no podemos olvidar que los alumnos están en periodo de aprendizaje, que todos pasamos por ello y que lo importante es aprender aunque no todos lo hagan al mismo ritmo.

\section{REFERENCIAS BIBLIOGRÁFICAS}

1. Smeltzer SC, Bare BG, Sholtis Brunner L, Smith Suddarth D. Manual de Enfermería Médico Quirúrgico. Estrés y Adaptación 8a Edición. México: Interamericana; 1998.

2. Papalia DE, Wendkos Olds S. Psicología. España: Mc Graw-Hill; 1995.

3. Mainieri R. Perspectiva cognitiva del estrés. J Soc Psychol 2008; 133 (2): 160-178.

4. Ticona Benavente BS, Paucar Quispe G, Llerena Callata G. Nivel de estrés y estrategias de afrontamiento en estudiantes de la Facultad de Enfermería - UNAS Arequipa. Enfermería Global 2006; 9 (19): 1-18. 
5. Bruno Selye H. Tensión sin angustia. Madrid: Guadarrama; 1975.

6. Cobo Cuenca Al, Carbonell Gómez de Zamora R, Rodríguez Aguilera C, Vivo Ortega I, Castellanos Rainero RM, Sánchez Donaire A. Estresares y ansiedad de los estudiantes de enfermería en sus primeras prácticas clínicas. NURE Inv [Revista en Internet] 2010 [07/03/2011]; 7(49): 1-13. Disponible en:

http://www.fuden.es/FICHEROS_ADMINISTRADOR/INV_NURE/NURE49_proyecto_e stresoresp.pdf

7. Holmes $\mathrm{TH}$, Rahe $\mathrm{RH}$. The social readjustment ratings scale. J Psychosom Res 1967; 11: 213-218.

8. Lázarus R, Folkman S. Estrés y procesos cognitivos. Barcelona: Martínez Roca; 1986.

9. Cano Vindel, A. Estrés laboral: la naturaleza del estrés. Sociedad española para el estudio del estrés $2002 . \quad$ Disponible en URL: http://www.ucm.es/info/seas/estrés lab/estrés.htm

10. Miguel Tobal, JJ. La ansiedad. Madrid: Santillana; 1996.

11. Pades Jiménez A, Homar Amengual C. El estrés de los estudiantes en las prácticas clínicas de enfermería. Rev Rol Enferm [Revista Electrónica] 2006 [27/02/2011]; $29 \quad$ (9): 19-24. Disponible en URL: http://dialnet.unirioja.es/servlet/articulo?codigo $=2107901$

12. Rodríguez Ramsbott GM. Efectos de un plan de técnicas de estudio en los estudiantes del primer semestre de la escuela de Enfermería [Tesis Doctoral]. Barquisimeto: Universidad Centro occidental Lisandro Alvarado, Decanato de Medicina; 1997.

13. López Medina I Mª , Sánchez Criado V. Percepción del estrés en estudiantes de enfermería en las prácticas clínicas. Enferm Clínica [Revista Electrónica] 2005 [14/0202011]; 15 (6): 307-313. Disponible en URL: http://www.elsevier.es/es/revistas/enfermeria-clinica-35/percepcion-estresestudiantes-enfermeria-las-practicas-clinicas-13082986-originales-2005

14. Juanola Pagés $M^{a} D$, Blanco Sánchez R, Cónsul Giribet M, Zapico Yáñez, F. Aprendizaje y satisfacción de los estudiantes de enfermería en las prácticas clínicas (II). Atención Primaria de Salud. Enferm Clín. 1998; 7 (1): 16-24.

15. Ferrer Pascual $M^{a} \mathrm{~A} A$, Rojo Pascual $M^{\mathfrak{a}} \mathrm{C}$, Ruiz Gómez $M^{\mathrm{a}} \mathrm{C}$, Fernández Araque $A M$, San Millán MG, Martínez León, JC. Análisis situacional de las prácticas clínicas en la Escuela de Enfermería de Soria. Metas Enferm. 2002; 5 (44): 18-22.

16. Sánchez M. Estrés de los estudiantes de enfermería en las prácticas clínicas. Enferm Cient. 1992; 127:43-46.

17. Zryewskyj T, Davis L. Sources of stress in third year baccalaureate nursing students. AARN-New-Lett. 1987; 43: 24-5

18. Zupiria Gorostidi X, Uranga Iturrioz Mã J, Alberdi Erize MJ, Barandiaran Lasa M. KEZKAK: Cuestionario bilingüe de estresores de los estudiantes de enfermería en las prácticas clínicas. Gac Sanit. 2003; 17 (1): 37-50

19. Timmins F, Kaliszer M. Aspects of nurse education programmes that frequently cause stress to nursing students- fact-finding sample survey. Nurse Educ Today. 2002; 22: 203-211.

20. Zupiria Gorostidi X, Uranga Iturrioz Mã J, Alberdi Erize MJ, Barandiaran Lasa M, Huitzi Egileor X, Sanz Cascante X. Fuentes de estrés en la práctica clínica de los 
estudiantes de enfermería: evolución a lo largo de la diplomatura. Enferm Clín. 2006;16 (5): 231-237.

21. Thomas JR, Nelson JK. Métodos de investigación en actividad física. Barcelona: Paidotribo; 2007.

22. Antolín Rodríguez R, Puialto Durán MJ, Moure Fernández ML, Quinteiro Antolín T. Situaciones en las prácticas clínicas que provocan estrés en los estudiantes de enfermería. Enfermería Global. 2007; 6 (1): 1-12.

23. López F, López MJ. Situaciones generadoras de estrés en los estudiantes de enfermería en las prácticas clínicas. Ciencia y Enferm. 2011; 2: 47-54.

24. Arribas Marín J. Toward a causal model of the dimensions of academic stress in nursing students. Rev Educ. 2011; (360): 533-556.

25. Amat Puig V, Fernández Gonzaga C, Orts Cortes I, Poveda Salva MR, Romá Ferri MT, Ribera Domene D. Estrés en estudiantes de enfermería. Rev ROL Enferm. 2008; 133: $75-80$. 